\title{
Automatic real-time detection of myocardial ischemia by epicardial accelerometer
}

Per Steinar Halvorsen, MD, ${ }^{a}$ Espen W. Remme, MSc, PhD, ${ }^{a}$ Andreas Espinoza, MD, ${ }^{a}$ Helge Skulstad, MD, $\mathrm{PhD},{ }^{\mathrm{b}}$ Runar Lundblad, MD, PhD,${ }^{\mathrm{c}} \mathrm{Jacob}$ Bergsland, MD, ${ }^{\mathrm{a}}$ Lars Hoff, MSc, PhD, ${ }^{\mathrm{d}}$ Kristin Imenes, MSc, $\mathrm{PhD},{ }^{\mathrm{d}}$ Thor Edvardsen, MD, PhD, ${ }^{\mathrm{b}, \mathrm{e}}$ Ole Jakob Elle, MD, PhD, ${ }^{\mathrm{a}, \mathrm{f}}$ and Erik Fosse, $\mathrm{MD}, \mathrm{PhD}^{\mathrm{a}, \mathrm{e}}$

\begin{abstract}
Objective: Myocardial ischemia may be detected with epicardial accelerometers. We developed and tested automated algorithms for real-time detection of myocardial ischemia by accelerometer measurements in both experimental and clinical settings.

Methods: In 10 pigs, an accelerometer was fixed to the epicardium in the area perfused by left anterior descending coronary artery. Acceleration and electrocardiogram were simultaneously recorded, and the QRS complex was automatically detected for exact timing of systole. Peak circumferential velocity and displacement were automatically calculated from epicardial acceleration signal within 150 milliseconds after peak $\mathrm{R}$ on electrocardiography. Global myocardial function was reduced by esmolol infusion, and regional function was altered by temporary left anterior descending occlusion. Automated ischemia detection analyses were tested in 7 patients during off-pump coronary artery bypass grafting. Left anterior descending coronary artery was occluded for 3 minutes before grafting. In both models, echocardiographic myocardial circumferential strain was used to confirm ischemia.
\end{abstract}

Results: Systolic displacement changed most during left anterior descending occlusion. Negative displacement during ischemia was found in pigs $(11.5 \pm 2.3$ to $-1.2 \pm 2.8 \mathrm{~mm}, P<.01)$; regional hypokinesia was found in clinical study $(12.8 \pm 8.1$ to $3.5 \pm 4.4 \mathrm{~mm}, P<.01)$. Ischemia was confirmed by echocardiography in both settings. Esmolol infusion induced smaller changes in automated accelerometer measurements than did left anterior descending occlusion $(P<.01)$.

Conclusions: Automatic real-time detection of myocardial ischemia with epicardial accelerometer was feasible. Automated ischemia detection analysis may be used for continuous monitoring of myocardial ischemia during cardiac surgery. (J Thorac Cardiovasc Surg 2010;139:1026-32)

Myocardial ischemia is associated with increased mortality after cardiac surgery. ${ }^{1}$ A sensor that continuously monitors and automatically detects ischemia during cardiac surgery would therefore be beneficial. Miniaturized accelerometers attached to the epicardium can provide real-time information of myocardial contraction. ${ }^{2-5}$ In our previous studies, an accelerometer improved the sensitivity of detection of ischemia relative to electrocardiography (ECG) and hemody-

From The Interventional Centre ${ }^{\mathrm{a}}$ and the Departments of Cardiology ${ }^{\mathrm{b}}$ and Cardiothoracic Surgery, ${ }^{c}$ Rikshospitalet University Hospital, Oslo, Norway; the Faculty of Science and Engineering, ${ }^{\mathrm{d}}$ Vestfold University College, Horten, Norway; and The Faculty of Medicine ${ }^{\mathrm{e}}$ and Department of Informatics, ${ }^{\mathrm{f}}$ University of Oslo, Norway.

Disclosures: P.S.H. was recipient of a clinical research fellowship from the Regional Health Authorities. A.E. was a recipient of a clinical research fellowship from the Norwegian Council of Cardio-vascular Diseases. The accelerometer is patented by Rikshospitalet University Hospital; O.J.E., E.F., and P.S.H. are patent holders.

Received for publication Feb 11, 2009; revisions received May 8, 2009; accepted for publication May 31, 2009; available ahead of print Aug 13, 2009.

Address for reprints: Per Steinar Halvorsen, MD, The Interventional Centre, Rikshospitalet University Hospital, NO-0027 Oslo, Norway (E-mail: per.steinar. halvorsen@rikshospitalet.no).

0022-5223/\$36.00

Copyright (c) 2010 by The American Association for Thoracic Surgery doi:10.1016/j.jtcvs.2009.05.031 namic monitoring. ${ }^{2-4}$ We have also demonstrated that systolic velocity in the circumferential direction in the left ventricle $(\mathrm{LV})$ is closely correlated with regional myocardial strain and with the maximum time derivative of $\mathrm{LV}$ pressure $\left(\mathrm{LV} \mathrm{dP} / \mathrm{dt}_{\max }\right)$. The accelerometer may therefore be used for monitoring of both regional and global LV function. Accelerometer signals were analyzed offline in our previous studies, but for clinical implementation automated analysis of accelerometer signals is necessary. The aim of this study was to develop a method for real-time automated detection of ischemia with signals from the accelerometer. On the basis of our previous experience, we hypothesized that (1) automated analysis of accelerometer systolic velocity and displacement curves may be used to detect regional myocardial ischemia and (2) automated analysis of epicardial systolic velocity and displacement may differentiate reduction in LV function induced by myocardial ischemia from global reduction induced by pharmacologic $\beta$-blockade.

\section{MATERIALS AND METHODS}

Accelerometer and Automated Analysis of Signals

A prototype 3-axis accelerometer with outer dimension of $11.0 \times 14.5 \times$ $5.2 \mathrm{~mm}$ (KXM52-1050; Kionix, Inc, Ithaca, NY) ${ }^{6}$ was sutured to the 


\section{Abbreviations and Acronyms \\ $\mathrm{CI}=$ confidence interval \\ ECG = electrocardiography \\ $\mathrm{LAD}=$ left anterior descending coronary artery \\ $\mathrm{LV}=$ left ventricle \\ $\mathrm{LV} \mathrm{dP} / \mathrm{dt}_{\max }=$ maximum time derivative of left ventricular pressure \\ ROC $=$ receiver operating characteristic}

anterior wall of the LV, distal to the second diagonal branch of the left anterior descending coronary artery (LAD; Figure 1). The accelerometer was sutured (5-0 Prolene; Ethicon, Inc, Somerville, NJ) to the epicardial layer, thereby limiting myocardial trauma. The 3-axis accelerometer allowed measurement of heart wall motion in three directions simultaneously. The axes of the accelerometer were aligned with the cardiac coordinate system. In this study, only circumferential acceleration was measured, because in the LV apical region circumferential contraction is most affected during ischemia. ${ }^{2-4}$ During systole, circumferential acceleration was defined positive in the counterclockwise direction, and epicardial velocity and displacement were calculated from the acceleration signal by single and double numeric integration. ${ }^{7}$ For exact timing of systole, ECG lead II was simultaneously recorded with the acceleration signal and the QRS complex automatically detected, as described by Hamilton and Tompkins. ${ }^{8}$ Epicardial peak systolic velocity within a 150 millisecond time interval after detection of the ECG R peak was then extracted and approximated midsystolic velocity (Figure 2). The displacement occurring during the 150-millisecond interval was automatically calculated and approximated midsystolic displacement (Figure 2). Experimentally, a criterion standard method of assessing regional LV work is the LV pressure-myocardial strain loop area as measured by sonomicrometry. ${ }^{9,10}$ In the evaluation of epicardial displacement for the assessment of LV function, we calculated the LV pressure-epicardial displacement loop area as a substitute.

Fluctuations in the epicardial accelerometer signal may occur from the effects of ventilation, similar to fluctuations found in arterial and venous pressure curves and in echocardiographic measurements. Ventilation rates during anesthesia are usually set between 10 and 20 ventilations/min in adults. To obtain reliable accelerometer estimates, the mean value of all heart beats in a 10-second interval was therefore calculated, ensuring that all heart beats in at least one ventilation cycle were included.

\section{Experimental Study}

The study was approved by the Rikshospitalet University Hospital institutional Animal Care and Use Committee. Ten Norwegian Landrace pigs, mean body weight $48 \pm 6 \mathrm{~kg}$, were anesthetized and surgically prepared as previously described in detail. ${ }^{4}$ Immediately after induction of anesthesia (intravenous pentobarbital $2-3 \mathrm{mg} / \mathrm{kg}$ and morphine $0.5 \mathrm{mg} / \mathrm{kg}$ ) a tracheostomy through a midline incision was performed. Anesthesia was maintained with inspired isoflurane $(1.0 \%)$ and an infusion of morphine $(0.15-0.2 \mathrm{mg} /$ $[\mathrm{kg} \cdot \mathrm{h}])$. A median sternotomy was performed, and the pericardium was split from apex to base. The chest and the pericardium were left open, and the pig was placed in the supine position.

Experimental protocol. After surgical preparation, the pig was allowed to recover for minimum 45 minutes. Global LV function was measured and changed by injection of esmolol $(100 \mathrm{mg})$. Maximal esmolol effect was recorded at nadir of LV pressure. After another 45 minutes of recovery, the LAD was occluded (duration, 2-3.5 minutes) and echocardiographic data were obtained. Echocardiographic recordings were initiated after a minimum of 1 minute of LAD occlusion. The LAD was subsequently kept occluded for another 20 to 30 seconds to achieve high-quality accelerometer data without interference from the ultrasound probe. Data were acquired at baseline and at each intervention.

Myocardial strain by speckle tracking echocardiogra-

phy. Strain is a measure of deformation of a material and in the heart reflects the myocardial contraction-relaxation pattern. By convention, negative strain is defined as shortening. In the circumferential direction, negative systolic strain represents the normal state, whereas positive systolic strain classifies lengthening and demonstrates the presence of severe myocardial ischemia. ${ }^{11}$ Peak systolic circumferential strain was used as a reference method to confirm ischemia during LAD occlusion. Strain was assessed in an apical LV short-axis slice by speckle tracking echocardiography (Vivid 7 scanner; GE Vingmed AS, Horten, Norway). Mean frame rate was $62 \pm 22$ frames/s. The speckle tracking method tracks the motion of the speckle pattern seen in the grayscale images from frame to frame through the cardiac cycle. The myocardial strain or deformation is calculated from the regional motion of the speckles.

Pressures and flow. LV pressure was assessed by a micromanometer (Millar Instruments, Inc, Houston, Tex). LV peak systolic and end-diastolic

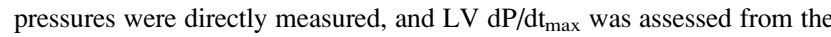
time derivative of the LV pressure. LAD flow was measured with a 4-mm ultrasonic transit time flow-probe (Medistim KirOp AS, Oslo, Norway). Pressure, acceleration, and ECG signals were recorded synchronously as previously described. ${ }^{4}$

\section{Clinical Study}

In a cohort of patients reported on previously, ${ }^{3}$ analyses of midsystolic velocity and midsystolic displacement were performed with the algorithms developed for the experimental study. A 3-minute pregrafting occlusion of the LAD was performed to induce ischemia. The occlusion was performed after at least 5 minutes of stable hemodynamic measurements and followed by at least 5 minutes of reperfusion. All patients in the cohort demonstrated ischemia on echocardiography during LAD occlusion ( $>30 \%$ reduction of myocardial shortening), whereas no significant changes in hemodynamics (heart rate, pulse contour cardiac output, and central venous pressure) and ECG were found. The regional ethics committee approved the patient study, and informed consent was obtained from all patients.

LV apical circumferential acceleration by accelerometer was continuously recorded, as described in the experimental model. Echocardiographic recordings were performed with a Vivid 7 scanner (GE Vingmed) with a transesophageal transducer. Apical short-axis projections were used to achieve similarity to the animal study. Circumferential systolic strain was measured in the apical LV anterior wall by speckle tracking analysis. Mean frame rate was $72 \pm 10$ frames/s.

\section{Statistical Analysis}

Parametric statistical methods were used, and all data are presented as mean $\pm \mathrm{SD}$ if not otherwise stated. In the experimental study, we used repeated measurements analysis of variance. All $P$ values were Bonferroni corrected for multiple comparisons. Receiver operating characteristic (ROC) curves were computed to determine cutoff values for optimal sensitivity and specificity. Standard table analysis was used to calculate positive and negative predictive values, and the $95 \%$ confidence intervals (CIs) were constructed with the $f$ distribution. For the correlation analysis, the Pearson correlation coefficient was computed. In the clinical study, paired data were compared with the Student $t$ test. The statistical analyses were computed with SPSS software (version 16; SPSS Inc, Chicago, Ill).

\section{RESULTS}

\section{Experimental Study}

LAD occlusion. There were significant decreases in accelerometer measurements of midsystolic velocity 


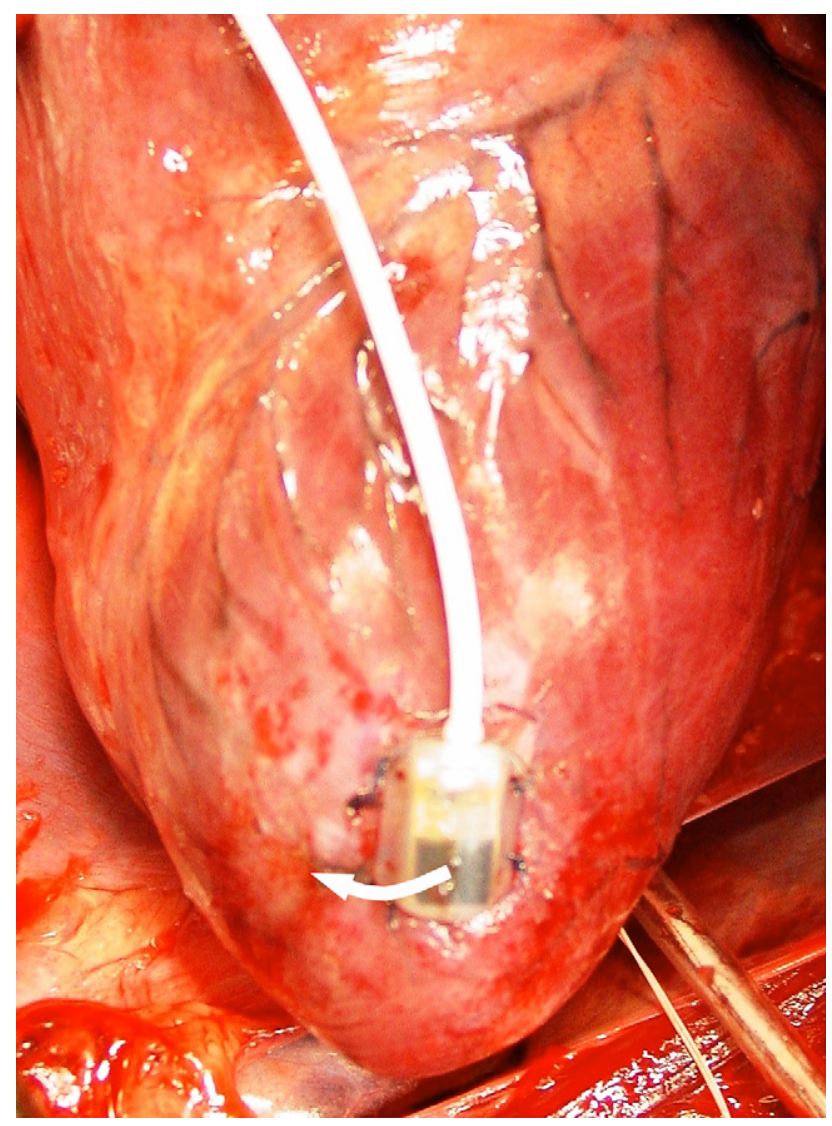

FIGURE 1. Anterior view of pig heart showing placement of 3-axis accelerometer in left anterior descending coronary artery perfusion area. Arrow indicates measured circumferential systolic motion.

$(13.2 \pm 2.8 \mathrm{~cm} / \mathrm{s}$ to $3.5 \pm 1.8 \mathrm{~cm} / \mathrm{s}, P<.01)$ and in LV pressure-displacement loop area $(1.84 \pm 0.60 \mathrm{~mm} \mathrm{Hg} \cdot \mathrm{m}$ to $0.23 \pm 0.31 \mathrm{~mm} \mathrm{Hg} \cdot \mathrm{m}, P<.01)$ during LAD occlusion. The greatest change in the accelerometer measurements, however, was found in midsystolic displacement, which demonstrated negative values during ischemia $(11.5 \pm 2.3$ $\mathrm{mm}$ to $-1.2 \pm 2.8 \mathrm{~mm}, P<.01$; Table 1 and Figures 2 and

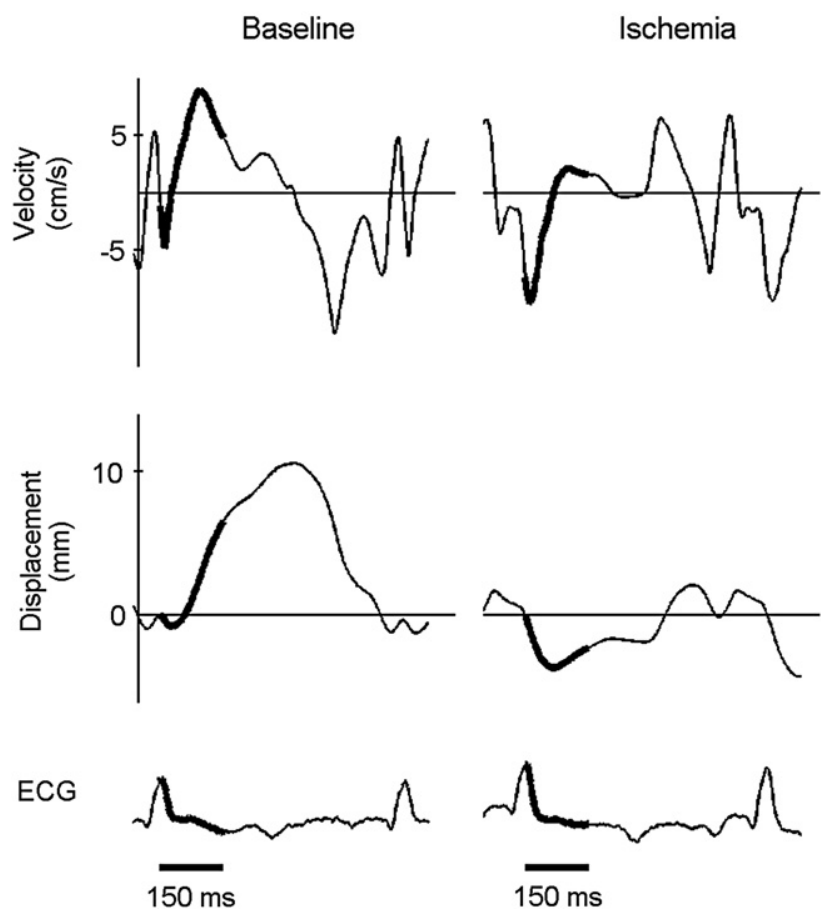

FIGURE 2. Experimental study. Accelerometer circumferential velocity and displacement curves are shown with electrocardiography $(E C G)$ at baseline and during left anterior descending coronary artery occlusion (Ischemia). Thick lines indicate automated 150-millisecond measurement interval after peak $\mathrm{R}$ on in which peak systolic velocity and displacement are assessed. In this interval, marked decreases in peak velocity and displacement are seen during occlusion, whereas ST segment is almost unchanged.

3). Negative midsystolic displacement values were observed in 7 of the 10 pigs during LAD occlusion. Correspondingly, negative midsystolic velocity and loop area were found in 1 pig. No negative values were found at baseline for the accelerometer variables.

Echocardiography. Regional circumferential strain according to echocardiography confirmed severe ischemia

TABLE 1. Experimental study: Accelerometer variables, echocardiographic strain, and hemodynamics during the interventions $(\mathbf{n}=10)$

\begin{tabular}{|c|c|c|c|c|}
\hline & Baseline & Esmolol & LAD occlusion & $P$ value* \\
\hline \multicolumn{5}{|l|}{ Accelerometer } \\
\hline Midsystolic velocity $150 \mathrm{~ms}(\mathrm{~cm} / \mathrm{s})$ & $13.2 \pm 2.8$ & $10.2 \pm 4.2$ & $3.5 \pm 1.8 \dagger \ddagger$ & $<.001$ \\
\hline $\mathrm{LV}$ pressure/displacement loop area $(\mathrm{mm} \mathrm{Hg} \cdot \mathrm{mm})$ & $1.84 \pm 0.60$ & $1.24 \pm 0.63 \dagger$ & $0.23 \pm 0.31 \dagger \S$ & $<.001$ \\
\hline Midsystolic displacement $150 \mathrm{~ms}(\mathrm{~mm})$ & $11.5 \pm 2.3$ & $7.2 \pm 4.6 \|$ & $-1.2 \pm 2.8 \dagger \ddagger$ & $<.001$ \\
\hline Echocardiographic systolic strain (\%) & $-26.4 \pm 5.9$ & $-20.4 \pm 4.3$ & $14.9 \pm 6.2 \dagger$ & $<.001$ \\
\hline Heart rate (beats/min) & $84 \pm 11$ & $82 \pm 9$ & $90 \pm 6 \ddagger$ & .016 \\
\hline LV peak systolic pressure $(\mathrm{mm} \mathrm{Hg})$ & $95 \pm 13$ & $75 \pm 15 \dagger$ & $83 \pm 16 \neq \|$ & $<.001$ \\
\hline $\mathrm{LV} \mathrm{dP} / \mathrm{dt}_{\max }(\mathrm{mm} \mathrm{Hg} / \mathrm{s})$ & $1569 \pm 334$ & $883 \pm 215 \dagger$ & $1306 \pm 290 \ddagger$ & .001 \\
\hline LV end-diastolic pressure $(\mathrm{mm} \mathrm{Hg})$ & $14 \pm 3$ & $14 \pm 3$ & $20 \pm 6 \S \|$ & .035 \\
\hline LAD flow $(\mathrm{mL} / \mathrm{min})$ & $23 \pm 14$ & $15 \pm 11 \|$ & $0 \pm 0 \S \|$ & .039 \\
\hline
\end{tabular}

Values are mean \pm SD. $P$ values are Bonferroni corrected. $L A D$, Left anterior descending coronary artery; $L V$, left ventricle; $L V d P / d t_{\max }$, maximum time derivative of left ventricular pressure. *Repeated measurements. $\dagger P<.01$ from baseline value. $\uparrow_{\uparrow} P<.001$ from esmolol. $\S P<.05$ from esmolol. $\| P<.05$ from baseline value. 


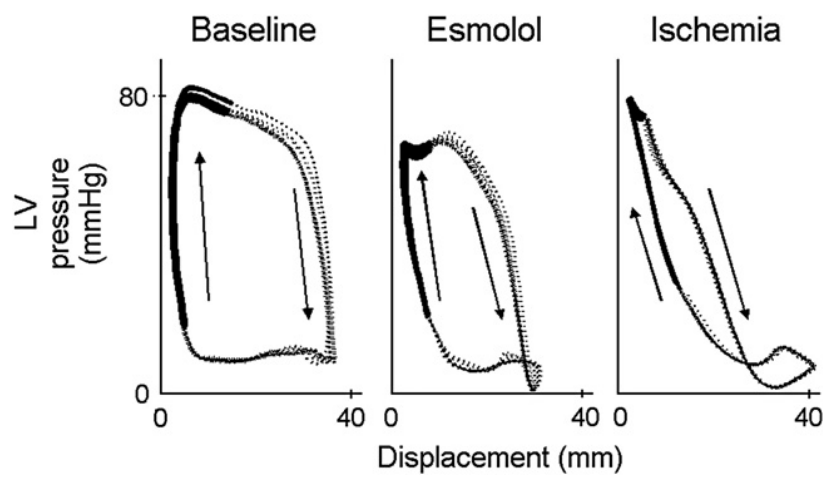

FIGURE 3. Experimental study. Left ventricular $(L V)$ pressure-accelerometer displacement loops are shown at baseline, with esmolol infusion, and with left anterior descending coronary artery occlusion (Ischemia). Loop area is decreased during esmolol infusion and further reduced during ischemia. Thick lines indicate systolic contraction in 150-millisecond interval from peak R on electrocardiography; arrows indicate loop direction.

(systolic lengthening) in all animals during LAD occlusion $(P<.01$; Table 1$)$.

Hemodynamic variables and ECG. Significant changes were observed during LAD occlusion in all hemodynamic variables except heart rate (Table 1). Ischemia decreased $\mathrm{LV}$ peak systolic pressure and $\mathrm{LV} \mathrm{dP} / \mathrm{dt}_{\max }$, whereas the LV end-diastolic pressure was increased, indicating reduced LV function. The ECG ST segment in lead II did not change significantly during LAD occlusion $(P=.519)$. One pig had ST-segment depression greater than $0.2 \mathrm{mV}$ during ischemia.

Intervention with esmolol. According to automated accelerometer measurements, midsystolic velocity, LV pressure-accelerometer displacement loop area, and midsystolic displacement all decreased during esmolol infusion, but the reductions were smaller than seen with LAD occlusion (all $P<.01$; Table 1 and Figure 4). No overlaps in data were seen between the intervention with esmolol and LAD occlusion for midsystolic velocity, whereas 2 observations for the loop area and 1 observation for midsystolic displacement in the esmolol data overlapped data obtained during ischemia.

No negative values for accelerometer midsystolic velocity, loop area, or midsystolic displacement were observed during the intervention with esmolol. With application of ROC analysis of baseline and interventional values, a cutoff value of $6.3 \mathrm{~cm} / \mathrm{s}$ for midsystolic velocity detected ischemia with sensitivity of $100 \%$ (95\% confidence interval [CI], $66 \%-100 \%)$ and specificity of $100 \%(95 \%$ CI, $80 \%-$ $100 \%$ ). For the LV pressure-displacement loop area, a cutoff value of $0.83 \mathrm{~mm} \mathrm{Hg} \cdot \mathrm{m}$ detected ischemia with sensitivity of $100 \%(95 \% \mathrm{CI}, 65 \%-100 \%)$ and specificity of $95 \%$ $(95 \%$ CI, $73 \%-100 \%)$. For midsystolic displacement, a cutoff value of $3.9 \mathrm{~mm}$ detected ischemia with sensitivity of $100 \%(95 \%$ CI, $66 \%-100 \%)$ and specificity of $90 \%$
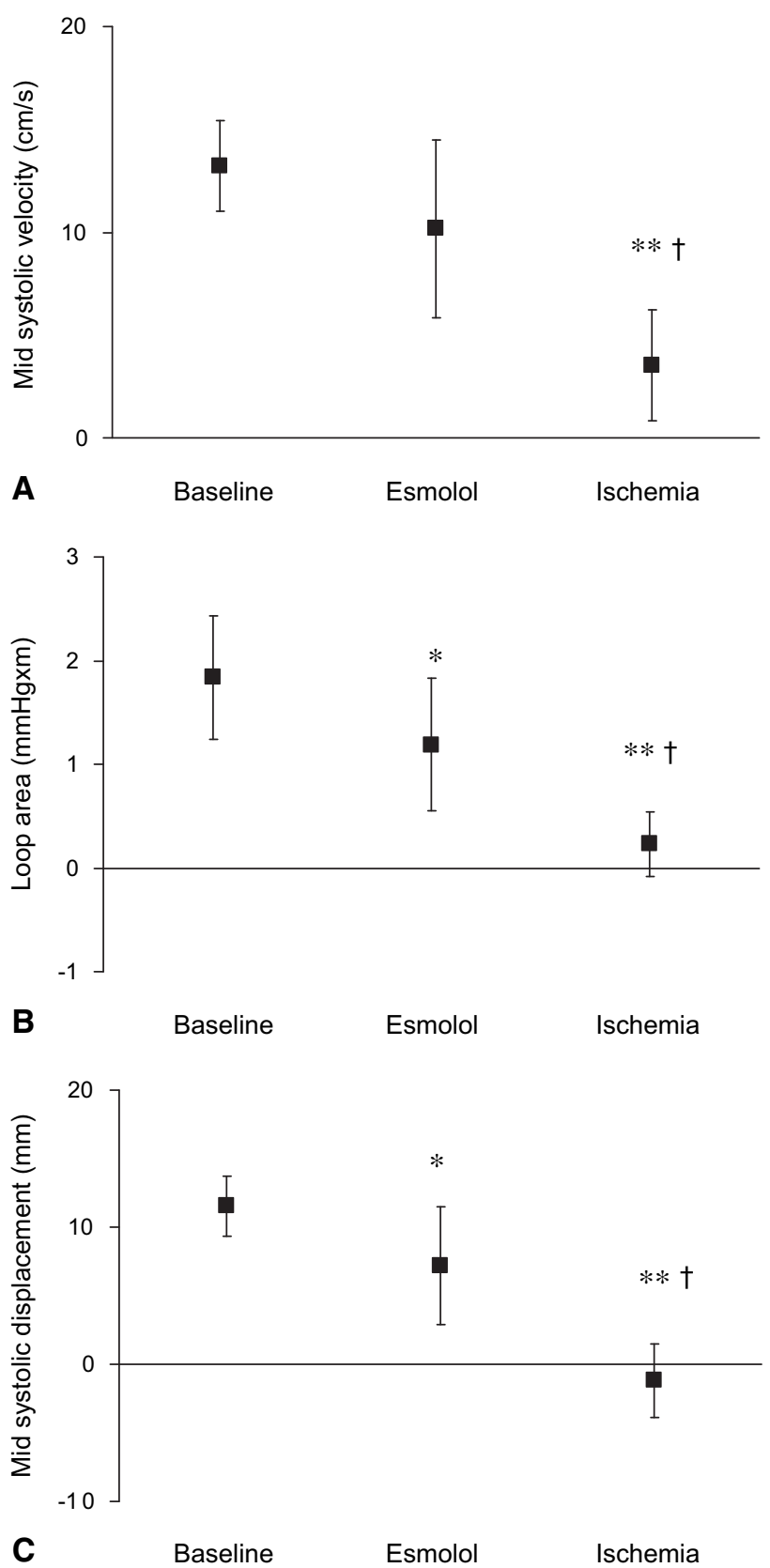

FIGURE 4. Experimental study. Measurements by accelerometer automated methods at baseline, esmolol infusion, and left anterior descending coronary artery occlusion (Ischemia). Greatest effect during ischemia can be seen in accelerometer-measured midsystolic displacement. Values are mean \pm SD. Asterisk indicates $P<.05$ from baseline; double asterisk indicates $P<.01$ from baseline; dagger indicates $P<.01$ from esmolol.

(95\% CI, 67\%-98\%). Positive predictive value for midsystolic velocity was $100 \%(95 \% \mathrm{CI}, 66 \%-100 \%)$, and negative predictive value was $100 \%(95 \% \mathrm{CI}, 80 \%-100 \%)$. For the loop area and midsystolic displacement, positive predictive values were $83 \%(95 \% \mathrm{CI}, 51 \%-97 \%)$ and $91 \%$ $(95 \%$ CI, 57\%-100\%), respectively, whereas negative 
predictive values were $100 \%(95 \% \mathrm{CI}, 78 \%-100 \%)$ and $100 \%$ (95\% CI, 79\%-100\%), respectively. All methods of analysis with the accelerometer therefore demonstrated the ability to discriminate between reduced myocardial function induced by ischemia and a reduction in global myocardial function induced by esmolol.

Midsystolic displacement was correlated closely with the LV pressure-displacement loop area $(r=0.90, P<.001)$.

Hemodynamic variables. Esmolol induced the greatest effect on global LV function, with LV pressure and LV $\mathrm{dP} / \mathrm{dt}_{\max }$ decreasing even more than during LAD occlusion (Table 1). No significant change in heart rate was seen during esmolol treatment, however, a significantly lesser effect than during LAD occlusion $(P<.01)$.

\section{Clinical Study}

We studied 7 patients ( 5 of them men) with a mean age of $62 \pm 8$ years, mean weight of $88 \pm 17 \mathrm{~kg}$, mean height of $173 \pm 10 \mathrm{~cm}$, median New York Heart Association functional class 2 (range, 1-3), mean LV ejection fraction $63 \% \pm 10 \%$, and mean LV end-diastolic pressure $11 \pm 4$ $\mathrm{mm} \mathrm{Hg}$. Two patients had histories of previous myocardial infarction, 1 in the right coronary artery perfusion area and 1 in the circumflex coronary artery perfusion area. Four had hypertension, 6 received $\beta$-blockers, and no patients had diabetes. All patients had significant stenosis of LAD with antegrade flow.

LAD occlusion. Ischemia induced marked changes in accelerometer epicardial midsystolic velocity $(12.6 \pm 5.9$ $\mathrm{cm} / \mathrm{s}$ to $6.5 \pm 2.8 \mathrm{~cm} / \mathrm{s}, P<.01)$ and epicardial midsystolic displacement $(12.8 \pm 8.1 \mathrm{~mm}$ to $3.5 \pm 4.4 \mathrm{~mm}, P<.01)$. Mean percentage change in accelerometer epicardial midsystolic velocity during LAD occlusion was $-48 \% \pm 63 \%$ (range, $-16 \%$ to $-76 \%$ ); that in epicardial midsystolic displacement was $-73 \% \pm 71 \%$ (range, $-23 \%$ to $-402 \%$ ). Reductions of more than $30 \%$ were found in 6 of 7 patients during ischemia by both accelerometer methods, implying a sensitivity of $86 \%$ (95\% CI, 42\%-99\%). Paradoxic movement in the LV apical region was found in 6 of 7 patients by strain echocardiography and in 2 of 7 patients by measuring accelerometer midsystolic displacement. No patients demonstrated negative accelerometer midsystolic velocities during ischemia. Thus, similar to what was found in the experimental study, the greatest clinical change in accelerometer measurements was observed in midsystolic displacement.

\section{DISCUSSION}

These experimental and clinical studies indicate that automated detection of myocardial ischemia by epicardial accelerometers is possible in real time with the described algorithms. Regional myocardial ischemia was detected with high sensitivity during LAD occlusion by measuring accelerometer midsystolic velocity and displacements. The automated accelerometer methods enabled discrimination of regional ischemia from a global reduction in LV function induced by esmolol. This technique offers promise as a potential monitor for ischemic events.

Exact timing of the accelerometer velocities and displacement in relation to systole is required to obtain analyzable data. This was achieved by simultaneous recording of ECG and automated detection of the ECG QRS complex. The rationale for measuring cardiac wall motions in the first part of systole is that ischemic segments shorten less or even stretch during early systole in coronary occlusion. ${ }^{12}$ The explanation for this is that most of the LV pressure generation occurs during early systole and that the ischemic segment behaves like a passive structure. Ischemia is also characterized by delayed systolic contraction, which typically starts in the second half of systole and lasts into the diastolic phase. ${ }^{13}$ One option for automatic detection of ischemic motion would be to analyze automatically deformation occurring during isovolumic relaxation. With limited measurements it is difficult to develop a robust, automatic method for detection of end-systole, however, whereas detection of the ECG $\mathrm{R}$ peak is more feasible. For these reasons we chose 150 milliseconds from the ECG R peak as our measurement interval.

Systolic velocity was included in the algorithms because previous work demonstrated the sensitivity of this parameter in detecting myocardial ischemia. ${ }^{3,4}$ Systolic displacement may be used in detection of ischemia by echocardiography. ${ }^{13}$ Displacement reflects the sum of all velocities in a time interval and thereby provides more information. Early systolic displacement incorporates velocity data from both isovolumic contraction and the beginning of ejection. The LV pressure-epicardial displacement loop area includes both epicardial systolic and diastolic displacements. This parameter usually cannot be used clinically, because LV pressure is not regularly measured. Our experimental data showed a strong correlation between midsystolic displacement and the LV pressure-epicardial displacement loop area, implying that the midsystolic displacement might be used as a surrogate for the loop area. Midsystolic displacement changed even more than did the loop area during coronary occlusion, indicating that the reduction in epicardial contraction during ischemia primarily occurs during early systole.

Myocardial ischemia is associated with increased mortality. ${ }^{1}$ Commonly applied methods, however, such as ECG and hemodynamic monitoring, may be insensitive in detecting ischemia. ${ }^{1,14}$ The accelerometer sensor is intended to function as an automatic ischemia detector, similar to ECG ST-segment monitoring. The automated accelerometer methods demonstrated the ability to detect myocardial ischemia and to discriminate ischemia from a global impairment in LV function. Accelerometers therefore have the potential to improve continuous ischemia detection in cardiac 
surgery. The technique offers promise for reducing perioperative myocardial infarctions, because the diagnosis and treatment of coronary artery occlusion may be performed earlier. The effect of treatment may also be guided more precisely by the accelerometer. Midsystolic displacement measured by the accelerometer demonstrated the greatest change during LAD occlusion, especially in the clinical study. Although the experimental and clinical data showed qualitatively similar changes, the differences were quantitatively different. This would be expected because of the difference in species. Also, the experimental animals had normal coronary arteries, whereas the patients had multivessel coronary artery disease.

Echocardiography, although an excellent tool to assess intraoperative ischemia, is cumbersome and not routinely applied for continuous monitoring after surgery. An automated system for real-time analysis of epicardial movement pattern may therefore become an important tool in postoperative monitoring of cardiac surgical patients. Additional miniaturization of the accelerometer will be essential for clinical implementation so that the device can be incorporated into temporary pacemaker leads. Such a sensor might be attached to the epicardium during surgery and withdrawn in the postoperative period, when the risk of ischemia is not longer imminent. Together with the automated ischemia detection method proposed here, a technique such as this could provide a beneficial peroperative and postoperative continuous monitoring system.

\section{LIMITATIONS}

The utility of accelerometer in ischemia monitoring needs to be demonstrated in larger clinical trials, with the described automated methods and blinding of the interpreters of the echocardiographic findings. Objectives in such a study would be to compare the methods for effectiveness in detecting intraoperative ischemia and to demonstrate fewer perioperative myocardial infarctions and reduced mortality when the device is used.

The duration of systole is relatively constant (300-350 milliseconds) during normal heart rate ranges but is significantly shortened during extreme tachycardia. An algorithm for adjustment of the systolic measurement interval relative to R-R duration should therefore be implemented in an automated monitoring device. Such a device should also have the capacity to adjust the sampling time for the averaging of heart beats. In the future, accelerometer measurements could be gated to respiration. This might improve positive and negative predictive values.

We have previously shown that accelerometer measurements in a remote nonischemic region were considerably affected during LAD occlusion because of tethering. This may indicate that a single sensor could detect abnormal motion caused by ischemia in other regions. This issue needs to be further investigated in a multivessel occlusion model.
Ischemia after surgery may not be related to graft patency alone but also to diffuse coronary artery disease. The accelerometer may not always be able to differentiate between these clinical situations. This limitation, however, is also seen with ECG and echocardiography. The automated accelerometer methods may not be as accurate during ventricular arrhythmias.

\section{CONCLUSIONS}

This study demonstrates the feasibility of real-time automated detection of ischemia with an accelerometer sensor. Both in animals and in patients undergoing coronary artery bypass grafting, automated analysis of accelerometer recordings demonstrated distinct changes during regional LV dysfunction. Experiments also indicate that algorithms based on the measurements from the accelerometer may discriminate between regional and global LV dysfunction. Accelerometers therefore have the potential to become important sensors for the detection of myocardial ischemia during and after cardiac surgery.

We thank statistician Milada Cvancarova Småstuen for valuable statistical assistance.

\section{References}

1. Comunale ME, Body SC, Ley C, Koch C, Roach G, Mathew JP, et al. The concordance of intraoperative left ventricular wall-motion abnormalities and electrocardiographic S-T segment changes: association with outcome after coronary revascularization. Multicenter Study of Perioperative Ischemia (McSPI) Research Group. Anesthesiology. 1998;88:945-54.

2. Elle OJ, Halvorsen S, Gulbrandsen MG, Aurdal L, Bakken A, Samset E, et al. Early recognition of regional cardiac ischemia using a 3-axis accelerometer sensor. Physiol Meas. 2005;26:429-40.

3. Halvorsen PS, Espinoza A, Fleischer LA, Elle OJ, Hoff L, Lundblad R, et al. Feasibility of a three-axis epicardial accelerometer in detecting myocardial ischemia in cardiac surgical patients. J Thorac Cardiovasc Surg. 2008;136: 1496-502.

4. Halvorsen PS, Fleischer LA, Espinoza A, Ell OJ, Hoff L, Skulstad H, et al. Detection of myocardial ischaemia by epicardial accelerometers in the pig. Br J Anaesth. 2009;102:29-37.

5. Wood JC, Festen MP, Lim MJ, Buda AJ, Barry DT. Regional effects of myocardial ischemia on epicardially recorded canine first heart sounds. J Appl Physiol. 1994;76:291-302.

6. Imenes K, Aasmundtveit K, Husa EM, Høgetveit JO, Halvorsen PS, Elle OJ, et al. Assembly and packaging of a three-axis micro accelerometer used for detection of heart infarction. Biomed Microdevices. 2007;9:951-7.

7. Hoff L, Elle OJ, Grimnes MJ, Halvorsen S, Alker HJ, Fosse E. Measurements of heart motion using accelerometers. Conf Proc IEEE Eng Med Biol Soc. 2004;3: 2049-51.

8. Hamilton PS, Tompkins WJ. Quantitative investigation of QRS detection rules using the MIT/BIH arrhythmia database. IEEE Trans Biomed Eng. 1986;33: 1157-65.

9. Forrester JS, Tyberg JV, Wyatt HL, Goldner S, Parmely WW, Swan HJ. Pressurelength loop: a new method for simultaneous measurement of segmental and total cardiac function. J Appl Physiol. 1974;37:771-5.

10. D'Ambra MN, Magrassi P, Lowenstein E, Kyo S, Austen WG, Buckley MJ, et al Myocardial temperature variation: effect on regional function and coronary flow in dogs. Am J Physiol. 1987;252(2 Pt 2):H448-55.

11. Amundsen BH, Helle-Valle T, Edvardsen T, Torp H, Crosby J, Lyseggen E, et al Noninvasive myocardial strain measurement by speckle tracking echocardiography: validation against sonomicrometry and tagged magnetic resonance imaging J Am Coll Cardiol. 2006;47:789-93. 
12. Edvardsen T, Aakhus S, Endresen K, Bjomerheim R, Smiseth OA, Ihlen H. Acute regional myocardial ischemia identified by 2-dimensional multiregion tissue Doppler imaging technique. J Am Soc Echocardiogr. 2000;13:986-94.

13. Skulstad H, Urheim S, Edvardsen T, Rein KA, Tønnessen TI, Hol PK, et al. Grading of myocardial dysfunction by tissue Doppler echocardiography: a comparison between velocity, displacement, and strain imaging in acute ischemia. $J$ Am Coll Cardiol. 2006;47:1672-82.

14. Gianrossi R, Detrano R, Mulvihill D, Lehmann K, Dubach P, Colombo A, et al. Exercise-induced ST depression in the diagnosis of coronary artery disease. A meta-analysis. Circulation. 1989;80:87-98. 